\title{
A Roof for Recovery: Combining the Principles of Housing First and Early Intervention in Services for People with Psychosis
}

\author{
Elizabeth Guevara \& Mireille Valois \\ Prevention and Early Intervention Program for Psychosis (PEPP-Montreal), Centre intégré \\ universitaire de santé et de services sociaux de l'Ouest-de-l'Île-de-Montréal \\ Ina Winkelmann \\ ACCESS Open Minds (pan-Canadian youth mental health services research network);Centre \\ intégré universitaire de santé et de services sociaux de l'Ouest-de-l'Île-de-Montréal \\ Ridha Joober \\ Prevention and Early Intervention Program for Psychosis (PEPP-Montreal); Douglas Hospital \\ Research Centre; Department of Psychiatry, McGill University \\ Karen Goldberg \\ Centre intégré universitaire de santé et de services sociaux de l'Ouest-de-l'Île-de-Montréal \\ Srividya N. Iyer \\ Prevention and Early Intervention Program for Psychosis (PEPP-Montreal); Douglas Hospital \\ Research Centre; Department of Psychiatry, McGill University
}

Elizabeth Guevara, Prevention and Early Intervention Program for Psychosis (PEPP-Montreal), Centre intégré universitaire de santé et de services sociaux de l'Ouest-de-l'T̂le-de-Montréal, Montreal, QC; Mireille Valois, Prevention and Early Intervention Program for Psychosis (PEPP-Montreal), Centre intégré universitaire de santé et de services sociaux de l'Ouest-de-l'Île-de-Montréal, Montreal, QC; Ina Winkelmann, Prevention and Early Intervention Program for Psychosis (PEPP-Montreal), Douglas Mental Health University Institute, Montréal, QC; Ridha Joober, Department of Psychiatry, McGill University; Douglas Hospital Research Centre; Prevention and Early Intervention Program for Psychosis (PEPP-Montreal), Montreal, QC; Karen Goldberg, Prevention and Early Intervention Program for Psychosis (PEPP-Montreal), Centre intégré universitaire de santé et de services sociaux de l'Ouest-de-l'Île-de-Montréal, Montreal, QC; Srividya N. Iyer, Department of Psychiatry, McGill University; Douglas Hospital Research Centre; Prevention and Early Intervention Program for Psychosis (PEPP-Montreal), Montreal, QC.

Ina Winkelmann is now also at ACCESS Open Minds (pan-Canadian CIHR-funded youth mental health services research initiative), Douglas Hospital Research Centre, Montreal, QC; Karen Goldberg is now the Centre intégré universitaire de santé et de services sociaux de l'Ouest-de-l'île-de-Montréal, Montreal, QC

Iyer and Joober have been supported through salary awards from the Fonds de Recherche du Quebec_Santé. Iyer also received a salary award from the Canadian Institutes of Health Research (CIHR). Iyer has received grants from CIHR. The authors would like 


\begin{abstract}
The availability of affordable, youth-friendly, recovery-oriented housing for persons with first-episode psychosis is limited. We report on the conceptualization, implementation and implications of a pilot housing project combining the principles of specialized early intervention for psychosis and those of "Housing First," which holds much promise to address this service gap.
\end{abstract}

Keywords: psychosis, early intervention, recovery, Housing First, employment

\title{
RÉSUMÉ
}

Les logements abordables, axés sur le rétablissement et adaptés aux jeunes atteints d'un premier épisode psychotique, se trouvent en nombre limité. Nous présentons ici un rapport sur la conceptualisation, la mise en œuvre et les incidences d'un projet pilote prometteur, combinant les principes de l'intervention précoce spécialisée pour la psychose et ceux du «Logement d'abord», susceptible de combler cette lacune dans les services.

Mots clés : psychose, intervention précoce, rétablissement, Logement d'abord, emploi

Youth with psychosis have similar housing-, education-, and employment-related objectives as their same-age peers (Rinaldi et al., 2010). Those among them who are homeless or in precarious housing situations need long-term, stable housing along with other supports and services to achieve early vocational and other functional recovery. The Mental Health Commission of Canada (2012) recognizes safe, affordable housing as essential to recovery. While research strongly supports the effectiveness of specialized early intervention for improving psychosis outcomes, little is known, particularly in Canada, about how such services can address their clients' housing needs. Our program innovation addresses this knowledge and practice gap.

\section{THE PEPP HOUSING PROJECT}

The Prevention and Early Intervention Program for Psychosis (PEPP) in Montreal provides two-year outpatient services to youth aged 14-35 with first-episode psychosis. Following international early intervention guidelines, PEPP offers recovery-oriented services led by case managers who provide sustained, intensive, one-on-one psychosocial care (Iyer, Jordan, MacDonald, Joober, \& Malla, 2015). While most PEPP clients live with family or in stable housing, some are homeless or precariously housed.

to thank the following individuals: Dr. Ashok Malla, Sonia Coté, Sherezad Abadi, Emily Meadows, Dr. Jai Shah, Amine Saadi and Chantal Fournier for their contributions to the administration of this project. We would like to thank Amparo Garcia for her initial support and encouragement of the ideas behind this project. We also thank all the participants of the PEPP-Montreal Housing Project. The Douglas Hospital Foundation has been instrumental in supporting the PEPP-Montreal Housing project by creating and managing a fund to which private donors contributed generously. This fund financed the subsidies provided towards participants' rent and the one-time furnishing grants given to each participant.

Correspondence concerning this article should be addressed to Srividya N. Iyer, PEPP-Montreal, Douglas Mental Health University Institute, 6875 LaSalle Blvd., Montreal, H4H 1R3. Email: srividya.iyer@mcgill.ca. 
Community housing facilities, even when intended for persons with mental illness, can be ill-suited to PEPP clients. Often, they are neither youth-friendly nor aligned with the early intervention philosophy that emphasizes developmentally normative community and vocational trajectories. Most of their residents are older and more persistently ill. For PEPP-type clients, living in such facilities may reinforce identification with the disorder and with "chronicity."

Further, many housing services require that residents receive disability payments, a condition that many PEPP clients do not meet. PEPP generally encourages clients to return to work or school, given their young age and the possible disincentivizing nature of disability payments. Many housing facilities' strict rules (such as no visitors and no alcohol) can destabilize housing tenure. Finally, there are long wait lists for general social housing and housing for individuals with mental illness.

In 2014, to address our clients' urgent need for more appropriate housing solutions, we created an innovative housing project combining early intervention for psychosis and the Housing First approach, both of which are evidence-based (Iyer et al., 2015; Tsemberis, Gulcur, \& Nakae, 2004).

Consistent with early intervention principles, a core component of the PEPP Housing Project is the sustenance of the phase-specific, recovery-focused services offered to all PEPP clients, including regular follow-up with case managers, medication (if agreed upon) and psychosocial interventions such as cognitivebehavioural therapy and family psychoeducation. All project participants are offered Individual Placement and Support (IPS; Becker \& Drake, 1994), an intervention backed by robust evidence for promoting vocational recovery among people with serious mental illness.

The PEPP Housing Project also follows the widely acknowledged core principles of the Housing First approach. This means that participants' access to housing is immediate and unconditional, e.g., not contingent on being in remission, employed/in school, substance-free or medication-adherent. Participants choose their apartment, neighbourhood and living arrangement (alone or with others). Housing is offered in the community, and not in a segregated facility or institution. The program's approach is recovery-oriented in its holistic view of well-being encompassing physical and mental health, economic independence and more. Services are tailor-made and responsive to individual participants' needs and preferences.

An occupational therapist conducts a detailed initial evaluation of participants' strengths and weaknesses vis-à-vis living skills and personal goals. Results from this evaluation are shared respectfully with participants and used to inform their housing choices and follow-up/support plans. Supports include home or community visits and the services of an occupational therapist-case manager and an employment specialist. Supports focus on a range of goals, including housing stability, mental health, life skills (such as budgeting), recreation and employment/education.

The housing program is offered to the few PEPP clients who are precariously housed or are awaiting discharge from the hospital but have no housing. The program lasts for two years beginning when participants enter it, independently of the point at which they are in PEPP's usual two-year protocol. Thus, clients entering the housing program late in their PEPP protocol can be followed for as long as four years.

Initially, participants contributed $25-30 \%$ of their monthly income towards rent and PEPP paid the remainder. Two years into the project's implementation, on participants' recommendation, this policy was revised to peg their contributions to their rent rather than their income. As preparation for eventually paying 
their full rent, participants now pay a progressively increasing proportion of their rent (up to 80\%). Participants receive an unconditional one-time grant for furniture and household items. The freedom to choose their furnishing is deemed critical to fostering participants' sense of autonomy, belonging, and being at home. With participants' consent, project staff also engage landlords.

Project staff (case managers, occupational therapist, employment specialist, and others) are full-time employees of PEPP's publicly funded parent institution. Rent and furnishing subsidies come from private donations to a Douglas Hospital Foundation fund. Donors have no influence on the project's design or execution.

\section{Preliminary Evaluation}

Between March 2014 and February 2018, 31 youth (24 male; 77\%) participated in the project. All participants were homeless or precariously housed at entry into the project. On average, participants entered the program aged 26.8 years (range $=18-36$ ) and having already received 17 months of treatment. All but four participants chose to live alone at entry. Of the four exceptions, one later opted to live alone. Four participants switched from living alone to living with others partway through their tenure in the project. Of the four who opted to live with others upon entry, one lived with a partner and child; one lived with a family member; and two lived with roommates.

Below, we report on some pertinent outcome domains:

(a) School/work integration: Ten participants (32\%) were working/studying at entry into the housing project and were offered IPS services to help maintain their employment/education. Nine of them remained engaged in work or school throughout their tenure in the project. The participant who could not maintain employment appears likely to resume working before completing the project. Of the 21 participants who were not working/studying at entry, 13 returned to work/school while enrolled in the project. Ten of these participants had received IPS services. Thus, 22 out of 31 participants (71\%) maintained or resumed employment/education. This is noteworthy given the usually low rates of vocational engagement in first-episode psychosis, even with specialized early intervention. Of the remaining nine participants, three are in the first six months of their tenure in the project and may well reintegrate into work/school later.

(b) Service engagement: Service engagement (such as attending appointments, collaborating with case managers, taking medications and/or participating in psychosocial interventions) is a key focus of early intervention in psychosis. Of the 31 participants, 11 (35.5\%) had disengaged from PEPP and were not attending appointments regularly when they entered the project. Enrollment in the housing project resulted in the re-engagement of all 11 in PEPP, indicating that stabilized housing provided the foundation for engagement in other interventions.

(c) Tenure: Of the 11 participants who have completed the project, six have chosen to continue living in their project-assigned apartments. Currently, 13 participants are in the project and seven have left it. Of those who left, three had difficulty living independently. One of these three moved to supervised housing and the other two moved in with their parents. One of those who left chose to return to a shelter and another ended up requiring hospitalization. Another of those leaving obtained government-subsidized housing and the last one disengaged completely from PEPP and the project. 
(d) Feedback from participants and landlords: Informal feedback was provided in writing by nine participants and six landlords and orally by several more. Participants and landlords expressed high levels of satisfaction with the project. Participants indicated that it promoted housing stability, increased autonomy and improved life skills. They reported desiring a longer duration of the project/subsidy, changes to the payment scheme (contributing a percentage of rent rather than income, a suggestion that was adopted), and increased support. Landlords reported high satisfaction with the project generally and with specific aspects like treatment team support and rent payments. They also reported positive changes in their perspectives about and more openness to individuals with mental health difficulties.

\section{Implications and Future Directions}

This project represents a model for combining early intervention for psychosis (with IPS included) and Housing First to offer stable housing to youth with psychosis and to promote their functional recovery and independence. Our experience indicates that this model is effective, likely cost-efficient, and acceptable to young service users, clinicians, and landlords.

Future challenges include securing sustainable funding for this program innovation. Efforts are also underway to integrate a peer support component and standardized outcome questionnaires into this project. Though tentative, our promising pilot project results indicate that a larger-scale study with appropriate funding and organizational support is warranted. Future research directions include a pragmatic cluster-randomized trial to evaluate the quality, outcomes and cost-effectiveness of our model in a larger early-psychosis sample. Such research is essential before our model is promoted widely as a viable option for early intervention services for persons with psychosis.

\section{REFERENCES}

Becker, D. R., \& Drake, R. E. (1994). Individual placement and support: A community mental health center approach to vocational rehabilitation. Community Mental Health Journal, 30(2), 193-206.

Iyer, S., Jordan, G., MacDonald, K., Joober, R., \& Malla, A. (2015). Early intervention for psychosis: A Canadian perspective. The Journal of Nervous and Mental Disease, 203(5), 356-364.

Mental Health Commission of Canada. (2012). Changing directions, changing lives: The mental health strategy for Canada. Calgary, AB: Author.

Rinaldi, M., Killackey, E., Smith, J., Shepherd, G., Singh, S. P., \& Craig, T. (2010). First episode psychosis and employment: A review. International Review of Psychiatry, 22(2), 148-162.

Tsemberis, S., Gulcur, L., \& Nakae, M. (2004). Housing first, consumer choice, and harm reduction for homeless individuals with a dual diagnosis. American Journal of Public Health, 94(4), 651-656. 